\title{
Analysis of the Influence of Knowledge and Belief of TCM on Behavior Based on Structural Equation Model
}

\author{
Jian $\mathrm{Wu}^{1}$, Xia Liu ${ }^{2}$, Junyong Wang ${ }^{1 *}$ \\ ${ }^{1}$ Institute of TCM and Health Development, Jiangxi University of TCM, Nanchang, Jiangxi, China \\ ${ }^{2}$ School of Basic Medical sciences, Jiangxi University of TCM, Nanchang, Jiangxi, China \\ Email : *wangjunyong2002@126.com
}

\begin{abstract}
Objective: To understand the influence of residents' knowledge and belief in TCM (TCM) on their behaviors, and to provide support for the government to optimize TCM policies; Methods: The structural equation model was used to analyze relation between TCM knowledge, belief and behaviors. Results: TCM knowledge had negative effects on TCM beliefs, and TCM beliefs strongly influenced TCM behaviors. Conclusion: the negative effect may be due to the influence of other factors between TCM knowledge and TCM belief; It is necessary to attach importance to the cultivation of TCM beliefs, strengthen the education and edification of TCM culture, give full play to the main role of various organizations and media, strengthen the standardization and supervision of TCM industry, and increase the input of TCM "inheritance and innovation".
\end{abstract}

Keywords: Traditional Chinese Medicine, Structural Equation Model, KABP

\section{INTRODUCTION}

Since the 18th CPC National Congress, the party and government have attached great importance to the development of TCM, and the development of TCM has ushered in a golden period.[1] TCM is an excellent traditional medicine in China, with its unique theoretical system and means of diagnosis and treatment, especially in disease prevention and rehabilitation. However, there are many problems in the development of TCM at this stage. Although there is a deep mass foundation, the proportion of Chinese medicine diagnosis and treatment behavior of the people in our country is low, [2] and the recognition

TCM knowledge, belief and behavior through empirical investigation and analysis, so as to provide theoretical research for expanding the influence of TCM and promoting residents' TCM diagnosis and treatment behavior.

\section{CONSTRUCTION OF THEORETICAL MODEL}

In order to understand the relationship among TCM knowledge, TCM belief and TCM behavior, a theoretical model was established based on KABP model.[3] Referring to relevant research, TCM knowledge can be divided into TCM knowledge, TCM knowledge and health care knowledge; TCM belief can be expressed as whether to believe in TCM, willingness to apply TCM knowledge, and willingness to recommend TCM; TCM behavior can be expressed as the frequency of accepting TCM service and recommending TCM knowledge.

\subsection{Model hypothesis}

Based on KABP, it can be assumed that TCM knowledge, TCM belief and TCM behavior are positively correlated. That is, knowledge strengthens belief, belief guides action, and knowledge promotes action.

\section{OBJECT AND METHOD}

\section{1. object}

The residents over 15 years old in Jiangxi Province have independent thinking and are willing to cooperate with the investigation. 


\section{2. method}

\subsection{1. $\quad$ sampling method}

The method of multi-stage stratified sampling was used to select 15 sample districts (counties) according to the East, West, South, North and provincial capital. Each district (county) was selected from 2 streets and 1 township (one of which was a county-level government resident); If there are no villages and towns in the survey area, one street is randomly selected to replace it, and two neighborhood committees are selected from each street (among them, one is the resident of the street office); Two village committees (one of which is the resident of township government) are selected from each township, and 100 permanent residents over 15 years old are selected from each district (county).

\subsection{2. investigation methods}

Face to face interviews were used to collect data.

\subsection{3. $\quad$ statistical methods}

Excel 2016 was used to establish a database for double data entry; Amos 22.0 was used for confirmatory factor analysis and structural equation model was established. [4]

\section{RESULTS}

\section{1. basic information}

A total of 1500 questionnaires were collected in this survey, and 1399 were effective, with an effective rate of $93.26 \%$. Among them, 642 (45.9\%) were male and 1628 $(54.1 \%)$ were female; The average age was 49.44 years old; Education level was mainly concentrated in junior high school $(33.10 \%)$, technical secondary school or senior high school (29.70\%), primary school and below (16.8\%); The non-agricultural population accounts for the majority $(66 \%)$ and the agricultural population accounts for less (34\%).

\section{2. model fitting}

According to the content of the questionnaire, the questions were divided into three latent variables: TCM knowledge, TCM belief and TCM behavior. The former is exogenous latent variable, and the latter two are endogenous latent variable. After modeling, estimation and correction by amos 22.0 software, the measurement models of 3 latent variables and 19 observed variables are finally obtained. The 19 observed variables are shown in Table 1 . The 13 observed variables of TCM knowledge can be divided into TCM knowledge, TCM knowledge and health care knowledge, and the division is shown in Table 3. The fitting results of the measurement model are $\mathrm{Cmin} / \mathrm{DF}=3.675, \mathrm{GFI}=0.988, \mathrm{NFI}=0.966, \mathrm{CFI}=$ $0.975, \mathrm{IFI}=0.975, \mathrm{RMR}=0.019, \mathrm{RMSEA}=0.044$, and the model fitting is acceptable. The path relationship after model modification is shown in Figure 1.

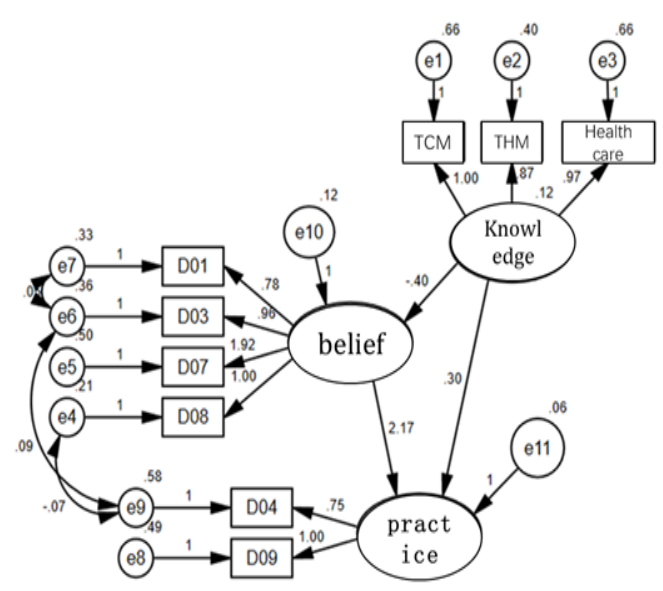

Fig. 1 transformation path diagram of TCM "KABP" SEM

Table 1. TCM health KABP SEM observation variable index

\begin{tabular}{|c|c|c|c|}
\hline \multicolumn{2}{|c|}{ classification } & number & Observed variables \\
\hline \multirow[t]{13}{*}{ Knowledge } & \multirow[t]{4}{*}{ TCM } & E28 & Thought and theory of TCM \\
\hline & & E30 & TCM diagnosis \\
\hline & & E32 & TCM Meridian Acupoints \\
\hline & & E35 & TCM Dietotherapy \\
\hline & \multirow{5}{*}{$\begin{array}{c}\text { Chinese } \\
\text { Herbal Medicine }\end{array}$} & E31 & Cognition of Chinese Herbal Medicine \\
\hline & & E33 & Concept of Chinese Herbal Medicine \\
\hline & & E36 & Efficacy of Chinese Herbal Medicine \\
\hline & & E39 & Methods of decocting and taking TCM \\
\hline & & E40 & TCM boiling apparatus \\
\hline & \multirow{4}{*}{ care ${ }^{\text {TCM Health }}$} & E29 & Cognition of TCM customs \\
\hline & & E34 & TCM fitness techniques \\
\hline & & E37 & Diet control of TCM \\
\hline & & E38 & Understanding of health care of medicine pillow \\
\hline \multicolumn{2}{|l|}{ belief } & D01 & Degree of belief in TCM \\
\hline
\end{tabular}




\begin{tabular}{|c|c|l|}
\hline \multirow{2}{*}{ practice } & D03 & Willingness to acquire knowledge of TCM \\
\cline { 2 - 3 } & D07 & Willingness to apply TCM knowledge \\
\cline { 2 - 3 } & D08 & Willingness to recommend TCM knowledge \\
\cline { 2 - 3 } & D04 & Frequency of receiving TCM services \\
\cline { 2 - 3 } & D09 & Frequency of recommending TCM knowledge \\
\hline
\end{tabular}

\subsection{Utility Analysis of Structural Model}

In this study, through the calculation of structural equation model, the relationship among residents' knowledge, belief and behavior of TCM is shown in Table 2 .

(1) The standardized coefficient of the direct effect is -0.359 , which means that when the residents' TCM knowledge is improved by 1 point, their TCM belief will be reduced by 0.359 points, which is inconsistent with the previous hypothesis.

(2) The standardized path coefficient of the direct effect is 0.993 , which means that the medical belief is improved by 1 point and the Chinese medicine behavior is improved by 0.993 points; Among them, the medical knowledge increased by 1 point, and the TCM behavior increased by 0.126 points, which is consistent with the hypothesis.
(3) TCM knowledge has a positive effect on TCM behavior, and the standardized path coefficient of direct effect is 0.126 , which means that TCM knowledge is improved by 1 point and TCM behavior is improved by 0.126 points, which is consistent with the hypothesis.

\section{4. result analysis of measurement model}

In the measurement model, there are three obvious variables loaded with TCM knowledge, and the factor loads are all greater than 0.381 , as shown in Table 4 . Among the three obvious variables, "TCM knowledge" has the greatest impact, followed by TCM knowledge, and finally health care knowledge; There were four significant variables loaded on TCM belief, among which the highest load of "willingness to apply TCM knowledge" was 0.719 , followed by "willingness to recommend TCM knowledge" with a load of 0.635; There are two significant variables that load the behavior of TCM, the factor load is greater than 0.63 , and the load of "frequency of recommending knowledge of TCM" is the highest, which is 0.762 .

Table 2. Coefficient Estimation Results

\begin{tabular}{|c|c|c|c|c|}
\hline Variables analyzed & S.E. & C.R. & $P$ & $\begin{array}{l}\text { Standardized path } \\
\text { coefficient estimation }\end{array}$ \\
\hline belief $\leftarrow$ Knowledge & 0.079 & -4.988 & $\star \star * *$ & -0.359 \\
\hline practice $\leftarrow$ belief & 0.124 & 17.49 & *** & 0.993 \\
\hline practice $\longleftarrow$ Knowledge & 0.132 & 2.292 & 0.022 & 0.126 \\
\hline TCM Knowledge & & & & 0.388 \\
\hline Chinese Herbal Medicine $\leftarrow$ Knowledge & 0.16 & 5.424 & *** & 0.427 \\
\hline TCM Health care $\leftarrow$ Knowledge & 0.179 & 5.442 & 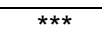 & 0.381 \\
\hline D08 belief & & & & 0.635 \\
\hline D07 belief & 0.099 & 19.356 & *** & 0.719 \\
\hline D03 belief & 0.063 & 15.368 & *** & 0.519 \\
\hline D01Łbelief & 0.056 & 14.025 & 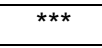 & 0.458 \\
\hline D09־practice & & & & 0.762 \\
\hline D04־practice & 0.041 & 18.254 & 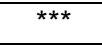 & 0.63 \\
\hline
\end{tabular}

Note: '***' means that the level of 0.01 is significant

\section{DISCUSSION}

\subsection{Discussion on the value of standardized path coefficient of latent variable}

In this study, there is a correlation between TCM knowledge, TCM belief and TCM behavior of residents in Jiangxi Province. TCM belief has the greatest impact on TCM behavior, and TCM knowledge has a negative effect on TCM belief.

\subsubsection{TCM knowledge and TCM belief have positive effects on TCM behavior.}

First, the highest value of the standardized path coefficient of TCM belief on TCM behavior is 0.993 , which indicates that TCM belief has a particularly significant impact on TCM behavior. In order to promote residents' TCM behavior, it is necessary to strengthen the cultivation of residents' TCM belief.

Second, the standardized coefficient of TCM knowledge on TCM behavior is only 0.126 , which 
indicates that the promotion effect of TCM knowledge level on TCM behavior is general. It may be that the TCM knowledge system is huge, the cognition of the masses is not comprehensive, the knowledge is not well applied, or even misused, which will certainly affect the diagnosis and treatment behavior of residents.

\subsubsection{Discussion on the negative effect of medical knowledge and TCM belief [5]}

One is the influence of modern medicine. Modern medicine and TCM belong to two different theoretical systems. The TCM system is huge, so it can't show us the principles and methods of disease prevention and treatment; The diagnosis and treatment methods of modern medicine make it easier for people to accept and understand, which leads to the decrease of residents' belief in TCM.

Second, in the process of dissemination of TCM knowledge, there are many swindlers in the grass-roots under the pretext of TCM name. They spread some of the most basic TCM knowledge, but they also seriously bruised the mass base of the survival and development of TCM, leading to the decline of residents' belief in TCM.

\subsection{Discussion on the relationship between explicit variables and latent variables}

First, among the latent variables of TCM knowledge, TCM knowledge has the greatest impact on TCM knowledge, and the standardized path coefficient is 0.427 , which indicates that in TCM knowledge, people are more willing to understand TCM knowledge, which may be that the effect of TCM on people is more direct and significant, and TCM is easier to apply in daily life.

Second, among the latent variables of TCM belief, the willingness to apply TCM knowledge has the greatest impact on TCM belief, and the standardized path coefficient is 0.719 , which indicates that the most intuitive performance of TCM belief is the willingness to apply TCM knowledge.

The third is the latent variable of TCM behavior. The frequency of TCM knowledge recommendation has the greatest impact on TCM behavior. The standardized path coefficient is 0.762 , mainly because some people are in good health, It does not necessarily need to accept TCM health services, but more shows the behavior of recommending TCM knowledge to others.

\section{CONCLUSION}

\subsection{Strengthen the construction of cultural atmosphere of TCM}

As the belief of TCM strongly affects the behavior of TCM and has a positive effect, it is necessary to strengthen the establishment and cultivation of the belief of TCM in order to strengthen the residents' behavior of TCM. [6] The cultivation of the belief of TCM can start from promoting TCM into schools and grass-roots units, creating a good atmosphere of TCM, and strengthening the trust and belief of the masses of TCM.

\subsection{Strengthen publicity and cultivate residents' belief in TCM}

With the development of the times, people's cognition and behavior are affected by many factors. The construction of Chinese medicine social atmosphere should not only give play to the authoritative advantages of traditional media, but also give play to the propaganda advantages of network platform and the characteristic advantages of Chinese medicine experience base. [7]

\subsection{Strengthen the regulation and supervision of TCM industry}

Strengthen the regulation and supervision of the TCM industry,[8] resolutely crack down on the organizations and individuals who cheat and falsely publicize under the guise of TCM, create a good organizational atmosphere and industry norms for the development of TCM, and prevent the swindler of TCM from continuously destroying the mass basis and social evaluation of TCM.

\subsection{Increase investment in "inheritance and innovation" of TCM}

Accelerate the inheritance and innovation of TCM, [9] give full play to the natural advantages of TCM in the prevention and treatment of diseases, rehabilitation and improving the quality of life. The second is to strengthen the development of science and technology of TCM, use modern science and technology, strengthen the standardized research and development of TCM, improve the dosage form of TCM, and improve the taste and taking way of TCM.

\section{ACKNOWLEDGMENT}

1. 2019 Jiangxi Province Humanities and Social Science Key Research Base Project (JD19067)

2. Jiangxi University of Traditional Chinese Medicine 1050 Talent Project Support Project

\section{REFERENCES}

[1] Xiao Xiao-He. Development of traditional Chinese medicine: New Era and new strategy. Zhong guo Zhong yao za zhi China journal of Chinese materia medica, 2019, 44(18) pp. 3837-3841.

[2] Min Deng. An empirical analysis of the willingness 
of Chinese residents to seek medical treatment in community based on social learning. China Health Statistics, 2015,32(03):pp.522-524.

[3] P. B. Lowry and J. Gaskin, "Partial Least Squares (PLS) Structural Equation Modeling (SEM) for Building and Testing Behavioral Causal Theory: When to Choose It and How to Use It," in IEEE Transactions on Professional Communication, vol. 57, no. 2, pp. 123-146, June 2014, doi: 10.1109/TPC.2014.2312452.

[4] Y. Wang, L. Cheng and S. Li, "Study on the Influence of Subway Passengers' Non-adaptive Behavior Based on Knowledge-Attitude-Practice Theory," 2019 International Conference on Intelligent Transportation, Big Data \& Smart City (ICITBS), 2019, pp. 72-76, doi: 10.1109/ICITBS.2019.00026.

[5] Du Suzhen et al. Discussion on the knowledge, belief, behavior intention of outpatients to traditional Chinese medicine and its related factors [C]. Chinese society of integrated traditional Chinese and Western medicine, Taiwan Changgeng Memorial Hospital. Proceedings of Cross Strait Symposium on Integrated Traditional Chinese and Western medicine. Chinese society of integrated traditional Chinese and Western medicine, Taiwan Changgeng Memorial Hospital: Chinese society of integrated traditional Chinese and Western medicine,2003, pp 63-64.
[6] J. Liu, X. Zhai, A. Han, S. Ding, et al. "The Future Development of Traditional Chinese Medicine from the Perspective of Artificial Intelligence with Big Data," 2018 IEEE 4th International Conference on Big Data Security on Cloud (BigDataSecurity), IEEE International Conference on High Performance and Smart Computing, (HPSC) and IEEE International Conference on Intelligent Data and Security (IDS), 2018, pp. 204-209, doi: 10.1109/BDS/HPSC/IDS18.2018.00051.

[7] H. Liang, J. Zheng, Q. Wang and Y. Chen, "Research on Development Strategy of "Internet +Traditional Chinese Medicine" Based on PEST-SWOT Model," 2020 International Conference on Public Health and Data Science (ICPHDS), 2020, pp. 232-236, doi: 10.1109/ICPHDS51617.2020.00052.

[8] Wei Li, Yuan Wang. The Existing Problems during the Further Development of Chinese Medicine Industry in Anguo. Information Engineering Research Institute, USA. Proceedings of 2012 2nd International Conference on Education and Education Management (EEM 2012 V3). Information Engineering Research Institute, USA, 2012: pp 253-255.

[9] Caiyun Peng et al. Research on the Integrating Practice of Professional Attainment and Cultural Inheritance of Traditional Chinese Medicine among College Students. Advances in Education, 2020, 10(02): pp 146-150. 